\title{
Habilidades psicológicas de coping em atletas brasileiros
}

\author{
Psychological coping skills in brazilian athletes
}

\author{
D.R. Coimbra, M. Bara Filho, A. Andrade, R. Miranda
}

ARTIGO ORIGINAL | ORIGINAL ARTICLE

\begin{abstract}
RESUMO
O objetivo do presente estudo foi analisar as habilidades de coping em atletas brasileiros. Especificamente, verificar as diferenças entre os sexos (masculino e feminino), esportes (coletivos e individuais), de diferentes níveis (regional nacional e internacional). A amostra foi de 375 atletas, idade média $16.8 \pm 2.1$. A maioria do sexo masculino $(74.7 \%)$ e de esportes coletivos $(68.3 \%)$. Em relação ao nível, 124 atletas foram classificados de nível regional, 72 nível nacional e 171 de internacional. O instrumento utilizado foi o Athletic Coping Skills Inventory-28 (ACSI-28), versão em português (ACSI-28BR) que aborda questões especificas de habilidades de coping no esporte. Houve diferença significativa para efeito principal na interação entre género, modalidade e nível competitivo para: Lidar com Adversidades e Desempenho sob Pressão. O estudo identificou diferentes contextos influenciam diretamente a aquisição de habilidades de coping.

Palavras-chave: habilidade de coping, desempenho, ACSI-28BR
\end{abstract}

ABSTRACT

The aim of the present study was to analyze the coping skills in Brazilian athletes. Specifically, verify the differences between the genders (male and female), sports (individual and collective), at different levels (regional, national and international). The sample was 375, mean age $16.8 \pm 2.1$, mostly male $(74.7 \%$ ) and sports collective $(68.3 \%)$. Concerning level, 124 athletes were rated regional level, 72 national level and 171 international level. The instrument used was the Coping Skills Inventory-28 (ACSI-28), Brazilian Portuguese version (ACSI-28BR) with items specific coping skills in the sport. Significant difference for main effect in the interaction between gender, sport and competitive level for: Coping with adversity and peak under pressure. The study found that different contexts directly influence the acquisition of coping skills.

Keywords: coping skill, performance, ACSI-28BR

Submetido: 05.03.2012 | Aceite: 27.12.2012

Danilo Reis Coimbra, Maurício Bara Filho, Renato Miranda. Faculdade de Educação Física e Desportos, FAEFID Universidade Federal de Juiz de Fora, UFJF - Juiz de Fora, MG. Brasil.

Alexandro Andrade. Centro de Ciências da Saúde e do Esporte, CEFID - Universidade do Estado de Santa Catarina, UDESC - Florianópolis, SC. Brasil.

Endereço para correspondência: Danilo Reis Coimbra, Faculdade de Educação Física e Desportos, Campus Universitário S/N - Martelos, CEP: 36036-900, Juiz de Fora, MG, Brasil.

E-mail: daniloreiscoimbra@yahoo.com.br 
Atualmente, o tema de maior repercussão no esporte é o estresse e a maneira como cada atleta lida com ele (Bebetsos \& Antoniou, 2003; Cresswell \& Hodge, 2004; Miranda \& Bara Filho, 2008; Nicholls \& Polman, 2007; Segato, Brandt, Liz, Vasconcellos, \& Andrade, 2010; Weinberg \& Gould, 2008). A este fenômeno de lidar com estresse denomina-se coping (enfrentar, lidar, gerenciar), ainda pouco estudado pelos psicólogos do esporte do Brasil e desconhecido da maioria dos atletas e treinadores (Gomes, Coimbra, Guillén, Miranda, \& Bara Filho, 2007). Lazarus e Folkman (1984) definem o coping como a capacidade de gerenciar os esforços cognitivos e comportamentais que estão constantemente modificando-se para administrar demandas psicofísicas específicas internas ou externas que excedem aos recursos individuais. (Anshel, 2001; Crocker, Kowalski, \& Graham, 1998).

Existem diferentes instrumentos que são utilizados para avaliar as habilidades psicológicas de coping em atletas (Crocker et al., 1998; Nicholls \& Polman, 2007). O Athletic Coping Skills Inventory-28 (ACSI-28) é um instrumento com 28 itens auto-preenchidos relacionados à percepção do atleta em situações típicas dos treinamentos e competições. Utilizando da Análise Fatorial Confirmatória e Exploratória, estabeleceram-se sete fatores (Lidar com Adversidades, Desempenho sob Pressão, Metas/Preparação Mental, Concentração, Livre de Preocupação, Confiança/Motivação, Treinabilidade). Cada um dos fatores é composto por quatro itens em uma escala tipo Likert de 0 (quase nunca) a 3 (quase sempre) e o escore dos 28 itens é denominado de Índice de Recursos Pessoais de Coping (Bebetos \& Antoniou, 2003; Goudas, Theodorakis, \& Karamousalidis, 1998; Smith \& Christensen, 1995; Smith, Schutz, Smoll, \& Ptacek, 1995).

Neste sentido, diversos grupos de pesquisas têm investigado as diferenças nas habilidades de coping medidas pelo ACSI-28 entre género, modalidade, nível competitivo, idade, etc.
(Bebetos \& Antoniou, 2003; Coetzee, Grobbelaar, \& Gird, 2006; Cresswell \& Hodge, 2004; Géczi et al., 2009; Goudas et al., 1998; Heever, Grobbelaar, \& Potgieter, 2007; Meyers, Stewarts, Laurent, Leunes, \& Bourgeois, 2008; Omar-Fauzee, Daud, Abdullah, \& Rashid, 2009). Smith, Schutz, Smoll, e Ptacek (1995) compararam atletas do género masculino e feminino e observaram que os do género masculino obtiveram maiores médias em cinco das sete dimensões (as mulheres obtiveram médias maiores em Treinabilidade e Livre de Preocupação). Bebetos e Antoniou (2003) abordaram as diferenças e semelhanças entre géneros e idades no preenchimento do instrumento. A análise demonstrou que não havia diferenças entre atletas homens e mulheres em nenhuma das dimensões. Goudas, Theodorakis, e Karamousalidis (1998) não encontraram diferenças significativas entre géneros em uma amostra de atletas de basquete masculino e feminino. Ambos apresentaram médias moderadas em todas as sete dimensões. Com atletas de diferentes modalidades e nível competitivo, Omar-Fauzee, Daud, Abdullah e Rashid (2009) encontraram diferenças estatisticamente significativas $(p<.05)$ entre os géneros apenas na dimensão Lidar com Adversidades (atletas masculinos obtiveram maior pontuação). No entanto, quando compararam atletas de diferentes níveis competitivos (regional, estadual e nacional), encontraram diferenças $(p<.05)$ nas sete dimensões do ACSI-28, sendo a média dos atletas nacionais maior em todas as escalas.

Pesquisas anteriores apontaram para a importância do coping no desempenho esportivo (Coetzee et al., 2006; Cox, Shannon, McGuire, \& McBride, 2010; Gould, Eklund, \& Jackson, 1993; Heever et al., 2007; Segato et al., 2010; Smith \& Christensen, 1995). Verificar o nível desta habilidade psicológica em atletas brasileiros, almejando a melhora destas aptidões, é um fator significativo para que o Brasil obtenha um bom desempenho em competições de alto nível. No entanto, há ainda carência de 
estudos de análise das habilidades psicológicas em atletas brasileiros. Os poucos já realizados encontraram diferenças significativas entre atletas do género masculino e feminino, entre modalidades e em diferentes níveis de desempenho na personalidade (Bara Filho, Ribeiro, \& Garcia, 2005) e nos motivos de início e abandono esportivo (Carmo, Matos, Ribas, Miranda, \& Bara Filho, 2009).

Pesquisas anteriores suportam a importância de verificar as diferenças nas habilidades psicológicas entre atletas de género, modalidade e nível competitivo distintos, a fim de traçar estratégias de intervenções para cada grupo, respeitando suas características e necessidades. Neste sentido, o objetivo do estudo foi analisar as habilidades de coping em atletas brasileiros. Especificamente, verificar as possíveis diferenças entre os géneros (masculino e feminino), modalidades (coletivas e individuais) e diferentes níveis (regional, nacional e internacional).

\section{MÉTODO}

\section{Amostra}

As características da amostra estão descritas na Tabela 1. A amostra foi composta de 375 atletas de diferentes níveis de desempenho, com idades entre 13 e 22 anos, tempo de prática entre um e 18 anos, sendo a maioria do género masculino $(74.7 \%)$, praticantes

Tabela 1

Características gerais da amostra

\begin{tabular}{cccc}
\hline Característica & $\begin{array}{c}\text { Feminino } \\
(n=95)\end{array}$ & $\begin{array}{c}\text { Masculino } \\
(n=280)\end{array}$ & $\begin{array}{c}\text { Total } \\
(n=375)\end{array}$ \\
\hline $\begin{array}{c}\text { Idade (anos) } \\
\text { Tempo de Prática } \\
\text { (anos) }\end{array}$ & $16.6 \pm 1.8$ & $17.0 \pm 1.9$ & $16.8 \pm 2.1$ \\
$\begin{array}{c}\text { Modalidade (Indi- } \\
\text { vidual/Coletiva) }\end{array}$ & $35 / 60$ & $84 / 196$ & $119 / 256$ \\
$\begin{array}{c}\text { Nível Competi- } \\
\text { tivo (Regional/ }\end{array}$ & $12 / 20 / 112 / 52 / 124 / 72 /$ \\
$\begin{array}{c}\text { Nacional/Interna- } \\
\text { cional) }\end{array}$ & 60 & 111 & 171 \\
\hline
\end{tabular}

Variáveis contínuas: Média \pm dp; variáveis categóricas: $n$ (número) de atletas por categoria de diferentes esportes (ténis, judo, basquete, volei, futebol, ginástica, etc.). As modalidades individuais representaram $31.7 \%$ da amostra $(n=119)$ e as modalidades coletivas $68.3 \%$ $(n=256)$. Quanto ao Nível Competitivo, 124 atletas foram classificados como nível regional (33.1\%), 72 como nível nacional (19.2\%) e 171 de nível internacional (45.6\%).

\section{Instrumentos}

Após realizar todos os procedimentos de adaptação transcultural (Beaton, Bombardier, Guillemin, \& Ferraz, 2002), utilizou-se a versão brasileira do Athletic Coping Skills Inventory-28 (ACSI-28BR). Cada uma das sete subscalas é composta por quatro itens, pontuados em uma escala tipo Likert ( $0=$ quase nunca até 3 = quase sempre), variando de 0 até 12 pontos. A soma de todas as subescalas é denominada Recurso Pessoal de Coping. As dimensões do ACSI-28BR foram definidas da seguinte forma: Lidar com Adversidades (ex: "Eu mantenho o controle emocional, não importa como as coisas estão indo comigo"), Desempenho sob Pressão (ex: "Minha tendência é jogar/ competir melhor sob pressão, pois eu penso mais claramente"), Metas/Preparação Mental (ex: "Eu tenho meu plano de competição completamente estruturado na minha mente muito antes de o jogo/competição começar"), Concentração (ex: "Para mim é fácil direcionar minha atenção e focar em um único objeto ou pessoa"), Livre de Preocupação (ex: "Eu me preocupo um pouco sobre o que as pessoas pensam sobre meu desempenho"), Confiança/ Motivação (ex: "Eu sinto confiante de que eu irei jogar/competir bem"), Treinabilidade (ex: "Eu aperfeiçôo minhas habilidades escutando cuidadosamente os conselhos e instruções dos técnicos e treinadores"). A versão brasileira do instrumento possui 28 itens e obteve Consistência Interna entre 0.54 (Treinabilidade) e 0.77 (Desempenho sob Pressão) e para a Escala Total o Alpha de Cronbach foi de 0.81. O escore $\alpha$ para algumas dimensões foi moderado, o que 
já era de se esperar, pois cada subescala possui apenas quatro itens e a amostra é bem ampla. No entanto, os valores de Alpha apresentados se assemelham com os reportados em outros estudos (Géczi et al., 2009; Goudas et al., 1998; Smith et al., 1995).

\section{Procedimentos}

Após a autorização para a realização do estudo, pelo Comitê de Ética em Pesquisa em Seres Humanos da Universidade Federal de Juiz de Fora (CEP-UFJF, parecer: 223/2009), e estando de acordo com os padrões exigidos pela Declaração de Helsinki (Resolução Conselho Nacional de Saúde 196/96) foi feito o contato com um grande clube da capital do Estado de Minas Gerais, que aloca diferentes modalidades. A coleta também foi realizada em competições regionais e nacionais, consentidas pelos organizadores, além da aplicação do questionário em diferentes equipes locais após a autorização do dirigente ou treinador.

Os atletas (ou responsáveis em caso de menores de 18 anos) assinaram o Termo de Consentimento Livre e Esclarecido (TCLE), informando-os a respeito dos objetivos do estudo, da garantia de anonimato quanto à identidade dos atletas e da participação voluntária na pesquisa. A duração para assinar o TCLE, preencher o questionário geral e específico variou entre 10' a 15'. A coleta foi realizada entre Novembro de 2009 e Abril de 2010.

\section{Análise Estatística}

Os dados foram analisados por meio da MANOVA $2 \times 2 \times 2$, para investigar a relação entre as habilidades psicológicas e o Nível Competitivo (regional x nacional/internacional) com possíveis efeitos do tipo de esporte (individual $\mathrm{x}$ coletivo) e género (masculino $\mathrm{x}$ feminino). Quando a análise multivariada detectou efeito significativo, efetuou-se a análise univariada por meio da ANOVA separadamente para cada variável dependente, ajustada pelo teste de Bonferroni. A análise da estatística $\mathrm{F}$ foi feita a partir do Traço de Pillai. Quando o pressuposto de esfericidade não foi assumido, foi usado o valor de ajuste fornecido pelo teste Greenhouse-Geiser. O Nível Competitivo, o tipo de modalidade esportiva e o género foram considerados as variáveis independentes, enquanto que as sete categorias de habilidades psicológicas de coping, as variáveis dependentes. Foram calculados os valores de cada uma das sete escalas (média $\pm \mathrm{dp}$ ). Para a variável Coping Total foi realizada uma ANOVA $2 \times 2 \times 2$ separadamente. As análises não foram ajustadas para a idade, em razão da não diferença significativa entre os grupos em função desta variável. Considerou-se um nível de significância $\alpha=0.05$ (Field, 2009).

\section{RESULTADOS}

A MANOVA $2 \times 2 \times 2$ resultou em efeitos principais significativos para género $[\mathrm{F}(7.366)=$ 8.31, $p=.000$; poder $=1.00]$, tipo de esporte $[\mathrm{F}(7.366)=2.41, p=.02$; poder $=0.86] \mathrm{e}$ nível competitivo $[\mathrm{F}(7.366)=4.06, p=.000$; poder $=0.98]$ bem como para as interações género*nível $[\mathrm{F}(7.366)=3.13, p=.003$; poder $=0.94]$, modalidade*nível $[\mathrm{F}(7.366)=1.98, p$ $=.05 ;$ poder $=0.77]$ e género*modalidade $*$ nível $[\mathrm{F}(7.366)=2.07, p=.04$; poder $=0.79] . \mathrm{A}$ Tabela 2 apresenta a média e o desvio-padrão das habilidades psicológicas para as categorias de género, nível competitivo e tipo de esporte. A Tabela 3 apresenta os valores da estatística $F$ da análise univariada para cada variável dependente.

\section{Quanto ao Género}

Em relação às habilidades psicológicas e o género das atletas, foram observados efeitos principais significativos para Melhor Desempenho sob Pressão, Lidar com Adversidades, Metas/Preparação Mental e Confiança/Motivação (Tabela 3). Independentemente do tipo de esporte e do nível competitivo, os atletas masculinos apresentaram maiores escores nestas habilidades psicológicas do que as 
atletas. Para Lidar com Adversidades, os atletas masculinos apresentaram ainda maiores valores tanto no nível regional quanto nacional (interação género*nível competitivo). Por outro lado, em Treinabilidade, as atletas apresentaram maiores valores no nível regional, ao passo que no nível nacional foram observados resultados similares para ambos.

Foram também encontrados efeitos de interações significativas para Concentração (género*tipo de esporte), Melhor Desempenho sob Pressão e Lidar com Adversidades (género*tipo de esporte*nível competitivo). As atletas do género feminino apresentaram maiores valores de Concentração nas modalidades individuais, enquanto que os atletas masculinos obtiveram maiores valores de Concentração nas modalidades coletivas. No nível regional, os atletas masculinos apresentaram maiores valores de Melhor Desempenho sob Pressão tanto nas modalidades individuais quanto nas coletivas quando comparados às atletas do género feminino. Já no nível nacional, este resultado foi observado somente nas modalidades coletivas, pois nas modalidades individuais, todos apresentaram valores similares.

\section{Quanto ao Tipo de Esporte}

Em relação às habilidades psicológicas e o tipo de esporte, foi encontrado efeito principal significante apenas para Treinabilidade (Tabela 3). Independentemente do género e do nível competitivo, atletas de esportes individuais reportaram maiores valores de Treinabilidade do que atletas de esportes coletivos. Observaram-se também efeitos de interações significativas para Concentração (género*tipo de esporte; tipo de esporte*nível competitivo). Os maiores valores de Concentração foram reportados por atletas de modalidades individuais e de nível regional. No nível nacional, foram observados valores similares para esportes individuais e coletivos. Houve interações significativas para Desempenho sob Pressão e Lidar com
Adversidades (género*tipo de esporte*nível competitivo). Os maiores valores de Melhor Desempenho sob Pressão foram observados em esportes coletivos masculinos de nível nacional, ao passo que os menores valores foram para esportes individuais femininos de nível regional. Quanto à variável Lidar com Adversidades, os maiores valores foram observados em atletas masculinos, sendo que no nível nacional, eles aparecem em esportes coletivos, ao passo que no nível regional os maiores valores ocorrem em esportes individuais.

\section{Quanto ao Nivel de Competitivo}

Em relação às habilidades psicológicas e o nível competitivo, foi encontrado efeito principal significante para Lidar com Adversidades, Metas/Preparação Mental, Livre de Preocupação, Treinabilidade e Confiança/Motivação (Tabela 2). Foram encontradas também interações significativas para Lidar com Adversidades e Treinabilidade (género*nível Competitivo) e Concentração (tipo de esporte*nível competitivo) e Melhor Desempenho sob Pressão e Lidar com Adversidades (género*tipo de esporte*nível competitivo).

Independentemente do género e do tipo de esporte, atletas de nível nacional apresentam maiores valores para Lidar com Adversidades, Metas/Preparação Mental, Livre de Preocupação e Confiança/Motivação, ao passo que atletas de nível regional apresentaram maiores valores de Treinabilidade. Os atletas masculinos reportaram valores similares de Lidar com Adversidades tanto os de nível regional quanto nacional, ao passo que as atletas femininas de nível nacional apresentaram maiores valores do que as de nível regional para esta variável. Quanto a Treinabilidade, valores similares foram observados em atletas masculinos de nível regional e nacional, sendo que nas atletas femininas, maiores valores de Treinabilidade foram observados no nível regional. Atletas de nível regional apresentaram maiores valores de Concentração em esportes individuais, 
Tabela 2

Média e desvio-padrão das habilidades psicológicas em função do Nível Competitivo, modalidade e gênero

\begin{tabular}{ccccccccc}
\hline & \multicolumn{4}{c}{ Atletas Masculino } & \multicolumn{3}{c}{ Atletas Feminino } \\
\hline & \multicolumn{1}{c}{ Esporte Individual } & Esporte Coletivo & Esporte Individual & Esporte Coletivo \\
\hline & Regional & Nacional & Regional & Nacional & Regional & Nacional & Regional & Nacional \\
& $n=19$ & $n=65$ & $n=97$ & $n=99$ & $n=7$ & $n=33$ & $n=13$ & $n=47$ \\
\hline Desempenho sob Pressão & $7.6 \pm 2.9$ & $6.6 \pm 3.0$ & $7.3 \pm 2.6$ & $8.5 \pm 2.7$ & $4.4 \pm 3.4$ & $6.6 \pm 3.4$ & $5.5 \pm 3.3$ & $5.9 \pm 3.1$ \\
Lidar com Adversidades & $8.0 \pm 2.2$ & $7.0 \pm 2.4$ & $7.2 \pm 2.2$ & $7.7 \pm 2.3$ & $3.8 \pm 2.9$ & $6.2 \pm 2.5$ & $5.2 \pm 2.2$ & $6.3 \pm 2.6$ \\
Metas/Preparação Mental & $8.0 \pm 3.0$ & $8.2 \pm 2.4$ & $7.1 \pm 2.4$ & $7.8 \pm 2.6$ & $5.8 \pm 3.1$ & $8.6 \pm 2.7$ & $6.1 \pm 2.3$ & $6.9 \pm 2.6$ \\
Livre de Preocupação & $3.8 \pm 2.7$ & $6.2 \pm 2.9$ & $5.4 \pm 2.7$ & $5.3 \pm 2.8$ & $5.3 \pm 3.7$ & $5.5 \pm 2.6$ & $4.6 \pm 2.4$ & $5.2 \pm 2.8$ \\
Concentração & $5.9 \pm 1.9$ & $5.5 \pm 2.0$ & $5.5 \pm 1.8$ & $6.2 \pm 1.9$ & $7.0 \pm 1.5$ & $5.6 \pm 2.1$ & $5.1 \pm 1.6$ & $5.4 \pm 1.8$ \\
Treinabilidade & $7.1 \pm 2.1$ & $7.2 \pm 1.7$ & $7.0 \pm 1.9$ & $6.8 \pm 1.8$ & $8.6 \pm 1.0$ & $7.0 \pm 2.0$ & $7.3 \pm 1.7$ & $6.5 \pm 2.0$ \\
Confiança/ Motivação & $7.0 \pm 1.5$ & $7.2 \pm 1.5$ & $6.8 \pm 1.6$ & $7.1 \pm 1.5$ & $5.0 \pm 2.4$ & $6.5 \pm 1.9$ & $5.8 \pm 1.9$ & $6.0 \pm 1.9$ \\
\hline
\end{tabular}

Variáveis contínuas: média \pm dp; variáveis categóricas: $n$ (número) de atletas por categoria

Tabela 3

Estatística $F$ da análise univariada

Habilidades Psicológicas

\begin{tabular}{cccccccc}
\hline Efeitos e Interações & $\begin{array}{c}\text { Desemp. } \\
\text { Pressão }\end{array}$ & Lidar & $\begin{array}{c}\text { Metas/Prep. } \\
\text { Mental }\end{array}$ & $\begin{array}{c}\text { Livre de } \\
\text { Preocupação }\end{array}$ & Concentração & Treinab. & $\begin{array}{c}\text { Confiança/ } \\
\text { Motivação }\end{array}$ \\
\hline Género (G) & $18.4^{* *}$ & $34.4^{* *}$ & $6.2^{* *}$ & 0.00 & 0.01 & 0.9 & $23.8^{* *}$ \\
Tipo de Esporte (TE) & 1.4 & 1.0 & 3.2 & 0.01 & 2.5 & $4.2^{*}$ & 0.00 \\
Nível Competitivo (NC) & 2.6 & $4.2^{*}$ & $8.2^{* *}$ & $3.7^{*}$ & 0.5 & $4.9^{*}$ & $5.3^{*}$ \\
G x TE & 0.5 & 1.2 & 0.00 & 0.9 & $4.3^{*}$ & 1.2 & 0.2 \\
G x NC & 1.8 & $7.3^{* *}$ & 2.9 & 0.8 & 1.5 & $4.4^{*}$ & 1.5 \\
TE x NC & 0.05 & 0.03 & 0.8 & 1.4 & $5.5^{*}$ & 0.2 & 1.2 \\
G x TE x NC & $5.3^{*}$ & $3.8^{*}$ & 2.3 & 3.0 & 0.2 & 0.7 & 1.9 \\
\hline
\end{tabular}

$\left({ }^{*} p<.05 ;{ }^{* *} p<.01\right)$

enquanto que atletas de nível nacional apresentaram maior Concentração nos esportes coletivos. Maiores valores de Melhor Desempenho sob Pressão e Lidar com Adversidades foram observados em atletas de nível nacional, principalmente em atletas masculinos e de esportes coletivos.

A Figura 1 e 2 ilustram graficamente as diferenças nas médias( \pm Erro Padrão), nas habilidades de coping Lidar com Adversidades e Desempenho sob Pressão, respectivamente. Foram as únicas que apresentaram interação significativa entre as três variáveis dependentes analisadas: Género, modalidade e nível competitivo.

\section{DISCUSSÃO}

A proposta desta investigação foi verificar as habilidades psicológicas de coping através do ACSI-28BR em atletas brasileiros, comparando as diferenças entre atletas do género masculino e feminino de modalidades coletivas e individuais e competindo em nível regional e nacional/internacional. Os resultados obtidos apontaram uma interação significativa nas três variáveis analisadas na dimensão Desempenho sob Pressão e Lidar com Adversidades. Concentração apresentou interação entre duas variáveis: Género e tipo de esporte e tipo de esporte e nível competitivo. Em Treinabilidade a interação ocorreu entre género e nível competitivo. As habilidades Metas/Preparação Mental (género e nível), Livre de Preocupação (nível) e Confiança/Motivação (género e nível), apesar de apresentarem diferenças nas variáveis individualmente, não mostraram interação.

Atletas masculinos de modalidades coletivas e nível nacional possuem os níveis mais elevados de Desempenho sob Pressão, seguido pelos atletas de modalidades individuais de nível regional e coletivo de nível regional, contrastando com as do género feminino de modalidades individuais e coletivas de nível regional. Em esportes coletivos, lidar com a 


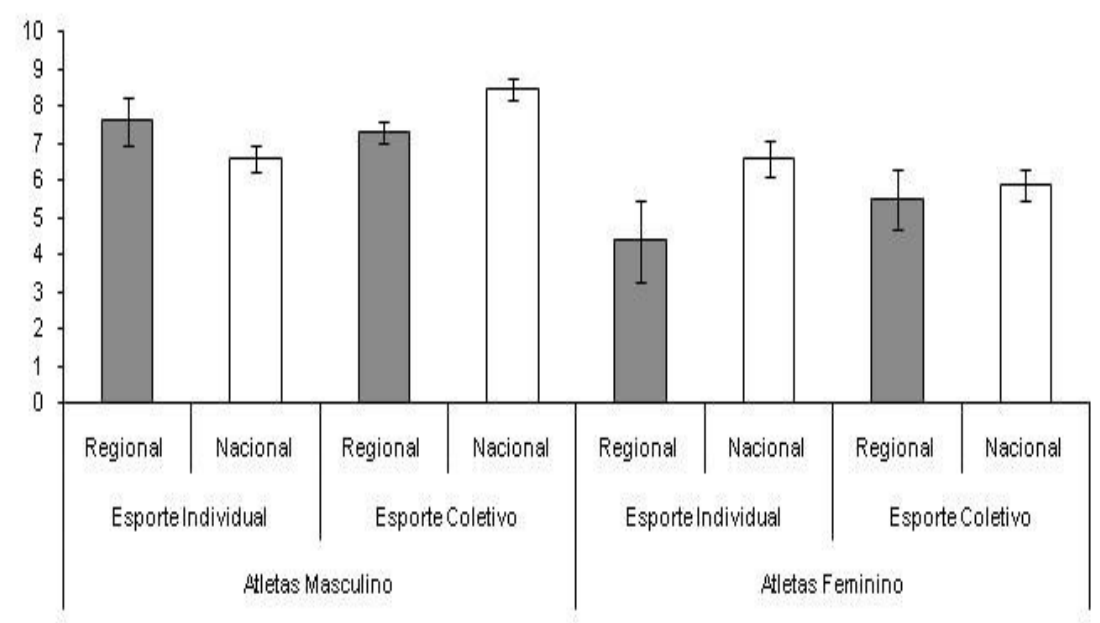

Figura 1. Diferenças $(\mathrm{M} \pm \mathrm{EP})$ na variável Desempenho sob Pressão

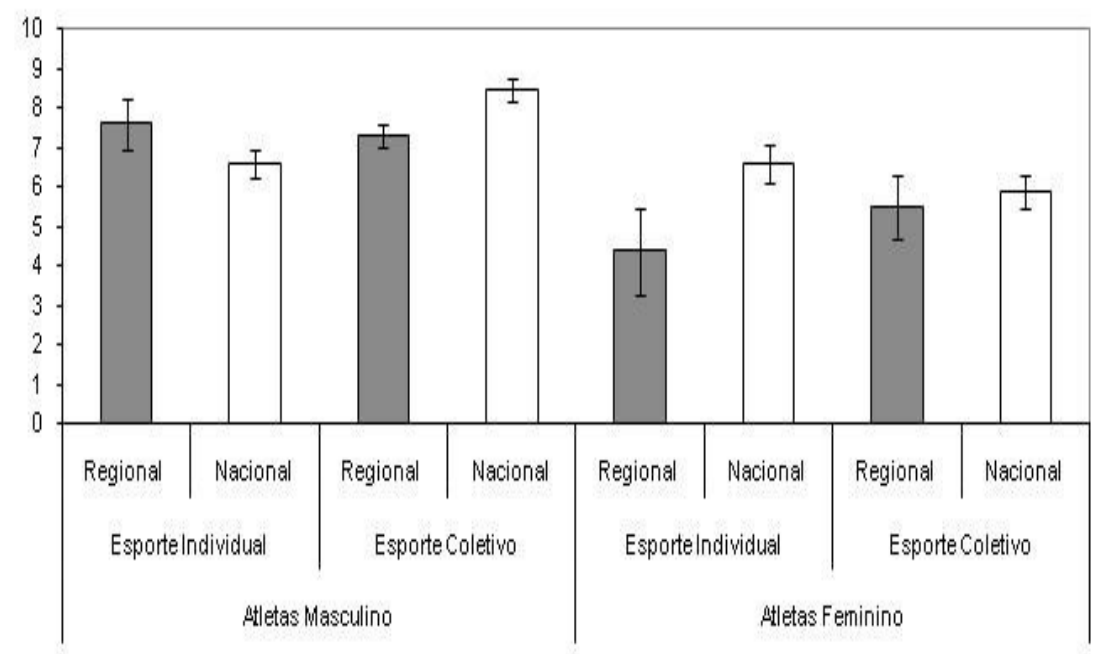

Figura 2. Diferenças $(\mathrm{M} \pm \mathrm{EP})$ na variável Lidar com Adversidades

pressão é uma tarefa equacionada por toda a equipe, há a possibilidade de substituição do atleta, geralmente possui maior duração e intervalos que servem para atenuar a situação, melhorando o desempenho, mesmo em situações adversas. No entanto, o que parece influenciar na habilidade de Desempenho sob Pressão é o género e não o esporte praticado. Lidar com adversidades é uma habilidade melhor desenvolvida em atletas masculinos de esportes individuais e nível regional, seguido pelos de modalidades coletivas e nível nacional. Mas, novamente, o que mais contribuiu para a diferença entre os grupos foi o género, embora a hipótese aqui seja de que atletas de moda- lidades coletivas lidam melhor com adversidades, pois, por serem esportes de confrontação direta e de habilidade motora aberta, ocorrem mais adversidades, mais situações inesperadas do que em atletas de esportes individuais, pois nestes geralmente não há a possibilidade de modificar um trajeto (por exemplo, atletismo), ou sair do percurso (por exemplo, natação), ou seja, são esportes mais previsíveis. Enfim, atletas de esportes coletivos por vivenciarem mais adversidades, poderiam desenvolver mais habilidades de enfrentá-las.

As diferenças encontradas entre os géneros nas habilidades de traçar metas e preparar-se mentalmente e, ainda, na confiança e motivação 
já é bastante difundida na literatura de psicologia do esporte (Weiberg \& Gould, 2008). Pesquisas anteriores apontam que atletas do género feminino possuem maiores níveis de ansiedade cognitiva e somática e menor autoconfiança, comparando com atletas do género masculino, bem como maior irritabilidade e emotividade, o que pode também influenciar em sua habilidade de desempenhar bem sob pressão e lidar com adversidades (Craft, Magyar, Becker, \& Feltz, 2003).

Diversas áreas do conhecimento buscam diferenciar homens e mulheres sob vários aspectos. No campo das ciências do esporte, encontrar essas diferenças entre o género é fundamental para a aplicação de diferentes métodos e meios de aplicação e avaliação para possibilitar o melhor rendimento individualizado (Bara Filho et al., 2005). Muitas vezes, o género do indivíduo é uma variável importante na relação entre habilidade psicológica e desempenho (Cox et al., 2010; Elferink-Gemser, 2005; Sheldon \& Eccles, 2005). No estudo de Bebetos e Antoniou (2003) foram encontrados valores similares nas dimensões do ACSI-28 entre homens e mulheres. No entanto é importante destacar que os atletas pesquisados eram de uma mesma equipe e treinadas pelo mesmo técnico, motivo apontado pelos autores como principal causa da ausência de diferenças entre os géneros. Smith et al. (1995) também não encontraram diferenças significativas entre homens e mulheres, bem como Goudas et al. (1998) com atletas de basquete. Ambos apresentaram médias moderadas em todas as sete dimensões e na escala total. Omar-Fauzee et al. (2009) encontraram diferenças estatisticamente significativas $(p<0,05)$ entre os géneros apenas na dimensão Lidar com Adversidades (atletas masculinos obtiveram maior pontuação), corroborando os achados do presente estudo. Weiberg e Gould (2008) e Miranda e Bara Filho (2008) sugerem diversos métodos que poderiam equacionar estas diferenças entre os géneros. Por exemplo, o uso de feedback positivo para aumentar a confiança e a motivação dos atletas, ou ainda, estratégias de ativação e desativação básica para obter um melhor desempenho mesmo sob pressão ou frente às adversidades. Traçar metas tangíveis a curto e médio prazo e o treinamento mental também são estratégias possíveis de serem ensinadas para melhorar as habilidades de preparação mental.

Sabendo das diferenças peculiares entre esportes coletivos e individuais, tanto em exigência física, técnica e metodológica, quanto em estrutura e preparação nos esportes (Bompa, 2002), características da personalidade (Bara Filho et al., 2005) humor (Beedie, Terry, \& Lane, 2000), habilidade psicológica (Cox et al., 2010; Elferink-Gemser, 2005) e desempenho optou-se por verificar também a diferença entre estas modalidades nas habilidades de coping. Atletas de modalidades individuais possuem maiores níveis de Treinabilidade do que atletas de esportes coletivos, independentemente de género e nível. Presume-se que esta diferença encontrada seja devida a parte da amostra ser composta por atletas de lutas. Notadamente nestes esportes há uma forte relação de subordinação ao treinador, inerente as características da modalidade há um maior respeito à experiência e ao conhecimento do mestre. Outro possível fator é que em esportes coletivos o treinador precisa equacionar a atenção para todos da equipe, diminuindo as chances de comunicação entre estes e todos os membros da equipe, além do caso de uma crítica ou correção ser dada na frente do time pode ser motivo para se envergonhar para alguns e sentir-se desafiados para outros.

$O$ tipo de modalidade também influenciou na habilidade de concentração. Embora os valores tenham-se mostrado próximos, aqueles de modalidade individual possuem esta habilidade mais desenvolvida do que os de coletiva, independentemente do género ou do nível competitivo. Ainda que em esportes como futsal, voleibol, basquete, etc. a exigência de 
se manter concentrado seja também elevada, nestas modalidades estímulos de distração são muito mais corriqueiros. Além do que, nestes esportes é difícil se manter concentrado do início ao fim de uma partida, pois como há a participação de todos da equipe, momentos de queda na concentração podem ocorrer sem que isto prejudique a equipe. No entanto, aquele atleta ou equipe que treina a concentração e mantém-se mobilizado do início ao fim de um jogo, possui grandes chances de obter um bom resultado (Miranda \& Bara Filho, 2008). Esportes individuais favorecem a concentração, pois durante uma competição o atleta necessita de concentrar em poucos focos de atenção. Porém caso este não obtenha um bom nível nesta habilidade, pode determinar a diferença entre o campeão e o último colocado.

A concentração também pareceu distinguir os atletas de modalidades coletivas e individuais no estudo de Elferink-Gemser (2005). Assim como no presente estudo atletas de esportes individuais apresentaram maiores níveis de concentração. As distrações estão mais presentes nos esportes coletivos e um ambiente mais calmo é esperado nos esportes individuais, embora ambos necessitem desenvolver bem esta habilidade, seja nos treinos ou em competições (Elferink-Gemser, 2005; Miranda \& Bara Filho, 2008; Weiberg \& Gould, 2008) para atingir um desempenho mais satisfatório. Atletas de níveis mais elevados obtiveram valores maiores de Concentração, amenizando inclusive a diferença entre os esportes. A tarefa de concentrar é sofisticada, no entanto deve ser treinada. $\mathrm{O}$ atleta deve ter em mente quais os focos de atenção ele necessita se concentrar, tanto em treinamentos quanto em competições. Miranda e Bara Filho (2008) apontam algumas diretrizes para melhorar o nível de concentração: Autoconhecimento, controlar interferências do meio ambiente, detectar detalhes com o máximo de atenção, táticas variadas de atuação, equacionar as emoções e estar bem condicionado fisicamente são algumas estraté- gias que podem ser ensinadas e treinadas desde o princípio.

Diversas pesquisas apontam para a importância das características psicológicas para o sucesso no alto rendimento. Avaliar essa relação tem sido objeto de diversos estudos na psicologia esportiva (Coetzee et al., 2006; Cox et al., 2010; Elferink-Gemser, 2005; Goudas et al., 1998; Smith \& Christensen, 1995). No presente estudo, o nível competitivo foi a variável onde mais diferenças entre os grupos foram encontradas. Todas as sete habilidades do ACSI-28BR, obtiveram diferenças estatísticas entre os níveis regional e nacional/ internacional, individualmente, em interação com o género, tipo de modalidade ou ambos, corroborando com o estudo de Goudas et al. (1998) com atletas gregos de basquete, atletas de nível nacional e internacional apresentaram maiores valores em Lidar com Adversidades e Confiança/Motivação. Cox et al. (2010) encontraram relação entre nível de desempenho e as habilidades psicológicas do ACSI-28, particularmente Confiança/Motivação e Livre de Preocupações. A relação entre a habilidade de traçar Metas/Preparação Mental e nível de desempenho também foi encontrada em atletas holandeses, corroborando o presente estudo, embora utilizasse instrumentos diferentes. Esta habilidade psicológica demonstrou diferença para atletas homens e mulheres, embora sem interação com o nível de desempenho (Elferink-Gemser, 2005).

Mesmo que os atletas de nível nacional tenham apresentado valores mais elevados para todas as dimensões, não se pode afirmar que exista uma relação de causa e efeito entre nível competitivo e habilidade psicológica. Ou seja, atletas de nível nacional desenvolvem melhor essas habilidades ou atletas com melhores habilidades alcançarão este nível competitivo. O que parece diferir atletas de nível regional dos de nível nacional e internacional é a quantidade e a qualidade das competições. Sabe-se que para um atleta desenvolver seu potencial 
psicofísico ele deve competir regularmente. Atletas que só competem em nível regional possuem menos chances de se aperfeiçoarem, pois enfrentam adversários com habilidades compatíveis. Já atletas que competem em nível nacional e internacional, além de participarem de mais competições, têm a possibilidade de enfrentar adversários de níveis de desempenho mais elevados, com habilidades distintas, levandos-o a desenvolver novas e aprimorar as suas capacidades psicofísicas.

$\mathrm{Na}$ realidade atual do esporte brasileiro, muitas vezes constata-se também atletas com potencial para competir em nível internacional, mas, devido à falta de estrutura competitiva e apoio para custos com inscrição, viagens, hospedagem, etc., estes atletas permanecem participando apenas de competições regionais. Embora a literatura aponte certa influência, não foi encontrada correlação com a idade nem com tempo de experiência em nenhuma das habilidades psicológicas aferidas pelo ACSI-28BR.

Por outro lado, o treinamento das habilidades psicológicas é necessário para qualquer género, esporte e nível competitivo, podendo ser aprendido ainda nos primeiros anos de prática. Por exemplo, a Treinabilidade, (habilidade de lidar bem com as exigências e orientações de treinadores), pode ser ensinada desde tenra idade. De acordo com Miranda e Bara Filho (2008), o pré-requisito básico para esta capacidade é a educação e boa comunicação entre técnicos e atletas, ou seja, é o atleta conseguir entender (e o treinador conseguir passar essa idéia) que, na maioria das vezes, uma orientação, uma cobrança, é feita no sentido de melhorar as habilidades gerais do atleta e não para ofendê-lo ou diminuí-lo. Apontam ainda, que os técnicos, principalmente os que lidam no esporte infanto-juvenil, deveriam desenvolver diversas competências essenciais para este trabalho, tais como: Conhecer aspectos específicos da modalidade, transmitir valores educativos, morais, sociais e culturais, trabalhar para que o atleta focalize o que fazer para vencer e não diretamente a vitória, ser bom modelo de comportamento e liderança para os jovens atletas. Geralmente nos esportes de rendimento, o atleta passa mais tempo com o treinador do que com a própria família, pois além de treinarem durante horas, viajam para competições, tornando uma relação muito próxima. Estudos que objetivam analisar a interação de mais de duas variáveis dependentes, como o caso do presente estudo, necessitam de um contingente amostral significativo para cada um dos subgrupos formados. Por isso, para atender esta exigência incluiu-se esportes não olímpicos, como futebol americano e jiu-jítsu. Do mesmo modo, atletas de nível internacional compõem o grupo de nível nacional. Ainda assim, houve um subgrupo com representação muito baixa: Atletas do género feminino de modalidades individuais e nível regional $(n=7)$. Por isso, os resultados deste grupo devem ser visto com cautela. Por ser um instrumento auto-preenchido, com itens que podem transmitir uma imagem projetada mais positiva do atleta, todas as dimensões do ACSI-28BR mostraram-se positivamente correlacionadas com a Escala de Desejo Social de Marlowe-Crowne (Smith et al., 1995). Todavia, esta relação pode ser diminuída se não houver nenhum dado que identifique o atleta no questionário, como foi o caso deste estudo. Por isso, é importante esclarecer que o ACSI-28BR não deve ser utilizado para selecionar atletas ou tomar qualquer outra decisão prática baseada no instrumento.

\section{CONCLUSÕES}

O estudo identificou que, de algum modo, diferentes contextos influenciam diretamente a aquisição de habilidades de coping em atletas brasileiros. Lidar com adversidades e ter um bom desempenho, mesmo em situação de pressão, são as habilidades que melhor distinguem esses contextos. Saber como esses atletas adquirem essas estratégias é fundamental para o esporte, no entanto, são necessárias mais pesquisas englobando amostras específicas 
maiores com o ACSI-28BR, para verificar como os atletas adquirem estas habilidades, a importância destes para o desempenho e se possuem relação com outras habilidades psicológicas.

\section{Agradecimentos:}

Nada declarado.

\section{Conflito de Interesses:}

Nada declarado.

\section{Financiamento:}

Nada declarado.

\section{REFERENCIAS}

Anshel, M. (2001). Qualitative validation of a model for coping with acute stress in sport. Journal of Sport Behavior, 24, 223-246.

Bara Filho, M., Ribeiro, L., \& García, F. (2005). Personalidade de atletas brasileiros de alto-rendimento: Comparações entre os gêneros masculino e feminino e correlação com nível competitivo e tempo de treinamento. Revista Portuguesa de Ciências do Desporto, 5(1), 31-39.

Beedie, C. J., Terry, P. C., \& Lane, A. M. (2000). The Profile of Mood States and Athletic Performance: Two meta-analyses. Journal of Applied Sport Psychology, 12(1) 49-68.

Beaton, D., Bombardier, C., Guillemin, F., \& Ferraz, M. (2002). Recommendations for the cross-cultural adaptation of health status measure. American Academy of Orthopedic Surgeons: Institute for Work and Health.

Bebetos, E., \& Antoniou, P. (2003). Psychological skills of Greek badminton athletes. Perceptual and Motor Skills, 97(3), 1289-1296.

Bompa, T. (2002). Periodização. (4 ${ }^{\mathrm{a}}$ Ed.). São Paulo: Phorte.

Carmo, J., Matos, F., Ribas, P., Miranda, R., \& Bara Filho, M. (2009). Motivos de início e abandono da prática esportiva em atletas brasileiros. $H U$ Revista, 35(4), 257-264.

Coetzee, B., Grobbelaar, H., \& Gird, C. (2006). Sport psychological skills that distinguish successful from less successful soccer teams. Journal of
Human Movement Studies, 51, 383-401.

Cox, R., Shannon, J., McGuire, R., \& McBride, A. (2010). Predicting subjective athletic performance from psychological skills after controlling for sex and sport. Journal of Sport Behavior, 33(2), 129-145.

Craft, L., Magyar, M., Becker, B., \& Feltz, D. (2003). The relationship between the competitive state anxiety inventory-2 and sport performance: A meta-analysis. Journal of Sport and Exercise Psycho$\log y, 25,44-65$.

Cresswell, S., \& Hodge, K. (2004). Coping skills: Role of trait sport confidence and trait anxiety. Perceptual and Motor Skills, 98(2), 433-438.

Crocker, P., Kowalski, K., \& Graham, T. (1998). Measurement of coping strategies in sport. In J. Duda. (Ed.), Advances in sport and exercise psychology measurement (pp. 149-161). Morgantown, WV: Fitness Information Technology.

Elferink-Gemser, M. (2005). Today's talented youth field hockey players, the stars of tomorrow? A study on talent development in field hockey. (Doctoral Dissertation), Groningen University, Holanda.

Field, A. (2009). Descobrindo a estatística usando o SPSS. ( ${ }^{\mathrm{a}}$ Ed). Porto Alegre: Artmed.

Géczi, G., Tóth, L., Sipos, K., Fügedi, B., Dancs, H., \& Bognár, J. (2009). Psychological profile of Hungarian national young ice hockey players. Kinesiology, 41 (1), 88-96.

Gomes, S., Coimbra, D., Guillén, F., Miranda, R., \& Bara Filho, M. (2007). Análise da produção científica em psicologia do esporte no Brasil e no exterior. Revista Iberoamericana de Psicología Del Ejercicio y El Deporte, 2(1), 25-40.

Goudas, M., Theodorakis, Y., \& Karamousalidis, G. (1998). Psychological skills in basketball: Preliminary study for development of a Greek form of the Athletic Coping Skills Inventory-28. Perceptual and Motor Skills, 86(1), 59-65.

Gould, D., Eklund, R., \& Jackson, S. (1993). Coping strategies used by U.S. Olympic wrestlers. Research Quarterly for Exercise and Sport, 64(1), 83-94.

Heever, Z., Grobbelaar, H., \& Potgieter, J. (2007). Sport psychological skills and netball perfor- 
mance. Journal of Human Movement Studies, 52, 109-124.

Lazarus, R., \& Folkman, S. (1984). Stress, appraisal and coping. New York: Springer.

Meyers, M., Stewarts, C., Laurent, M., Leunes, A., \& Bourgeois, A. (2008). Coping skills of Olympic developmental soccer athletes. International Journal of Sports Medicine, 29(12), 987-993.

Miranda, R., \& Bara Filho, M. (2008). Construindo um atleta vencedor: Uma abordagem psicofísica do esporte. Porto Alegre: Artmed.

Nicholls, A., \& Polman, R. (2007). Coping in sport: A systematic review. Journal of Sport Sciences, 25 (1), 11-31.

Omar-Fauzee, M., Daud, W., Abdullah, R., \& Rashid, S. (2009). The effectiveness of imagery and coping strategies in sport. European Journal of Social Sciences, 9(1), 97-108.

Segato, L., Brandt, R. Liz, C., Vasconcellos, D., \& Andrade, A. (2010). Estresse psicológico de velejadores de alto nível esportivo em competição. Motricidade, 6(3), 53-62.
Sheldon, J., \& Eccles, J. (2005). Physical and psychological predictors of perceived ability in adult male and female tennis players. Journal of Applied Sport Psychology, 17(1), 48-63.

Smith, R., \& Christensen, D. (1995). Psychological skills as predictors of performance and survival in professional baseball. Journal of Sport and Exercise Psychology, 17, 399-415.

Smith, R., Schutz, R., Smoll, F., \& Ptacek, J. (1995). Development and validation of a multidimensional measure of sport-specific psychological skills: The Athletic Coping Skills Inventory-28. Journal of Sport and Exercise Psychology, 17, 379-398.

Weinberg, R., \& Gould, D. (2008). Fundamentos da psicologia do esporte e do exercício. ( $\left.3^{\mathrm{a}} \mathrm{Ed}\right)$. Porto Alegre: Artmed.

(cc) EY-No Todo o conteúdo da revista Motricidade está licenciado sob a Creative Commons, exceto quando especificado em contrário e nos conteúdos retirados de outras fontes bibliográficas. 